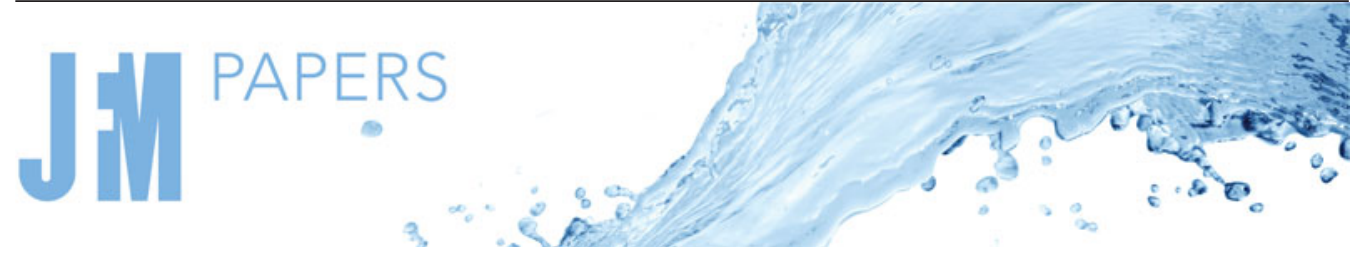

\title{
The effect of Prandtl number on turbulent sheared thermal convection
}

\author{
Alexander Blass ${ }^{1} \uparrow$, Pier Tabak ${ }^{1}$, Roberto Verzicco ${ }^{1,2,3}$, \\ Richard J.A.M. Stevens ${ }^{1}$ and Detlef Lohse ${ }^{1,4, \dagger}$ \\ ${ }^{1}$ Physics of Fluids Group, Max Planck Center for Complex Fluid Dynamics, J. M. Burgers Center for \\ Fluid Dynamics and MESA+ Research Institute, Department of Science and Technology, University of \\ Twente, P.O. Box 217, 7500 AE Enschede, The Netherlands \\ ${ }^{2}$ Dipartimento di Ingegneria Industriale, University of Rome "Tor Vergata", Via del Politecnico 1, \\ Roma 00133, Italy \\ ${ }^{3}$ Gran Sasso Science Institute, Viale F. Crispi, 7, 67100 L'Aquila, Italy \\ ${ }^{4}$ Max Planck Institute for Dynamics and Self-Organization, Am Fassberg 17, 37077 Göttingen, Germany
}

(Received 6 July 2020; revised 29 September 2020; accepted 10 November 2020)

In turbulent wall sheared thermal convection, there are three different flow regimes, depending on the relative relevance of thermal forcing and wall shear. In this paper, we report the results of direct numerical simulations of such sheared Rayleigh-Bénard convection, at fixed Rayleigh number $R a=10^{6}$, varying the wall Reynolds number in the range $0 \leqslant R e_{w} \leqslant 4000$ and Prandtl number $0.22 \leqslant P r \leqslant 4.6$, extending our prior work by Blass et al. (J. Fluid Mech., vol. 897, 2020, A22), where $\operatorname{Pr}$ was kept constant at unity and the thermal forcing $(R a)$ varied. We cover a wide span of bulk Richardson numbers $0.014 \leqslant R i \leqslant 100$ and show that the Prandtl number strongly influences the morphology and dynamics of the flow structures. In particular, at fixed $R a$ and $R e_{w}$, a high Prandtl number causes stronger momentum transport from the walls and therefore yields a greater impact of the wall shear on the flow structures, resulting in an increased effect of $R e_{w}$ on the Nusselt number. Furthermore, we analyse the thermal and kinetic boundary layer thicknesses and relate their behaviour to the resulting flow regimes. For the largest shear rates and $\operatorname{Pr}$ numbers, we observe the emergence of a Prandtl-von Kármán log layer, signalling the onset of turbulent dynamics in the boundary layer.

Key words: turbulent convection, Bénard convection, atmospheric flows

†Email addresses for correspondence: a.blass@utwente.nl,d.lohse@utwente.nl

(C) The Author(s), 2021. Published by Cambridge University Press. This is an Open Access article, distributed under the terms of the Creative Commons Attribution licence (http://creativecommons.org/ licenses/by/4.0/), which permits unrestricted re-use, distribution, and reproduction in any medium, provided the original work is properly cited. 


\section{A. Blass and others}

\section{Introduction}

Buoyancy and shear are crucial processes in fluid dynamics and key for many flow related phenomena in nature and technology. A paradigmatic example of buoyancy driven flow is Rayleigh-Bénard (RB) convection, a system where the fluid is heated from below and cooled from above (Ahlers, Grossmann \& Lohse 2009; Lohse \& Xia 2010; Chilla \& Schumacher 2012; Xia 2013). The flow is controlled by the Rayleigh number $R a=\beta g H^{3} \Delta /(\kappa \nu)$, which quantifies the non-dimensional thermal driving strength between the two horizontal plates. Here, $H$ is their distance, $\beta$ the thermal expansion coefficient of the fluid, $g$ the gravitational acceleration, $\Delta$ the temperature difference across the fluid layer, $\kappa$ and $v$ the thermal diffusivity and kinematic viscosity, respectively. Furthermore, the Prandtl number is defined as $\operatorname{Pr}=v / \kappa$, which is the ratio between momentum and thermal diffusivities. An important output of the flow is the heat transport between the plates, which can be non-dimensionally quantified by the Nusselt number $N u=Q H /(\kappa \Delta)$, with $Q=\langle w T\rangle_{A, t}-\kappa\left\langle\partial_{z} T\right\rangle_{A, t}$ the mean vertical heat flux, where $\langle\cdots\rangle_{A, t}$ indicates the mean over time and a horizontal plane.

On the other hand, for flows driven by wall shear stress, a commonly used model problem is the Couette flow (Thurlow \& Klewicki 2000; Barkley \& Tuckerman 2005; Tuckerman \& Barkley 2011). We adopt a geometry in which the bottom and top walls slide in opposite directions with a wall-tangential velocity $u_{w}$ and the forcing can be expressed non-dimensionally by the wall Reynolds number $R e_{w}=H u_{w} / v$. The relevant flow output is now the wall friction, quantified by the friction coefficient $C_{f}=2 \tau_{w} /\left(\rho u_{w}^{2}\right)$, with $\rho$ the fluid density and $\tau_{w}$ the surface- and time-averaged wall shear stress. Turbulent Couette flow is dominated by large-scale streaks (Lee \& Kim 1991; Tsukahara, Kawamura \& Shingai 2006; Kitoh \& Umeki 2008; Pirozzoli, Bernardini \& Orlandi 2011, 2014; Orlandi, Bernardini \& Pirozzoli 2015; Chantry, Tuckerman \& Barkley 2017). These remain correlated in the streamwise direction for a length up to approximately 160 times the distance between the plates (Lee \& Moser 2018).

Combining the buoyancy and wall shear forcings yields a complex system that is relevant in many applications, especially for atmospheric and oceanic flows (Deardorff 1972; Moeng 1984; Khanna \& Brasseur 1998). Also, in sheared thermal convection, large-scale structures emerge, as experiments have shown (Ingersoll 1966; Solomon \& Gollub 1990). Investigations on channel flows with unstable stratification (Fukui \& Nakajima 1985) revealed that temperature fluctuations in the bulk decrease while velocity fluctuations close to the wall increase for stronger unstable stratification.

Numerical simulations of wall sheared convection (Hathaway \& Somerville 1986; Domaradzki \& Metcalfe 1988) have revealed that adding shear to buoyancy increases the heat transport for low $R a$, but also causes the large-scale structures to weaken, thus decreasing the heat transport for $R a \gtrsim 150.000$. Similar phenomena have been observed in Poiseuille-RB, where the wall parallel mean flow is driven by a pressure gradient rather than the wall shear: in this case, the $N u$ decrease was attributed to the disturbance via the longitudinal wind of the thermal plumes (Scagliarini, Gylfason \& Toschi 2014; Scagliarini et al. 2015; Pirozzoli et al. 2017). This plume-sweeping mechanism, causing a Nusselt number drop, was also observed in Blass et al. (2020), who report very long thin streaks, similar to those of the atmospheric boundary layer where these convection rolls are called cloud streets (Etling \& Brown 1993; Kim, Park \& Moeng 2003; Jayaraman \& Brasseur 2018). 

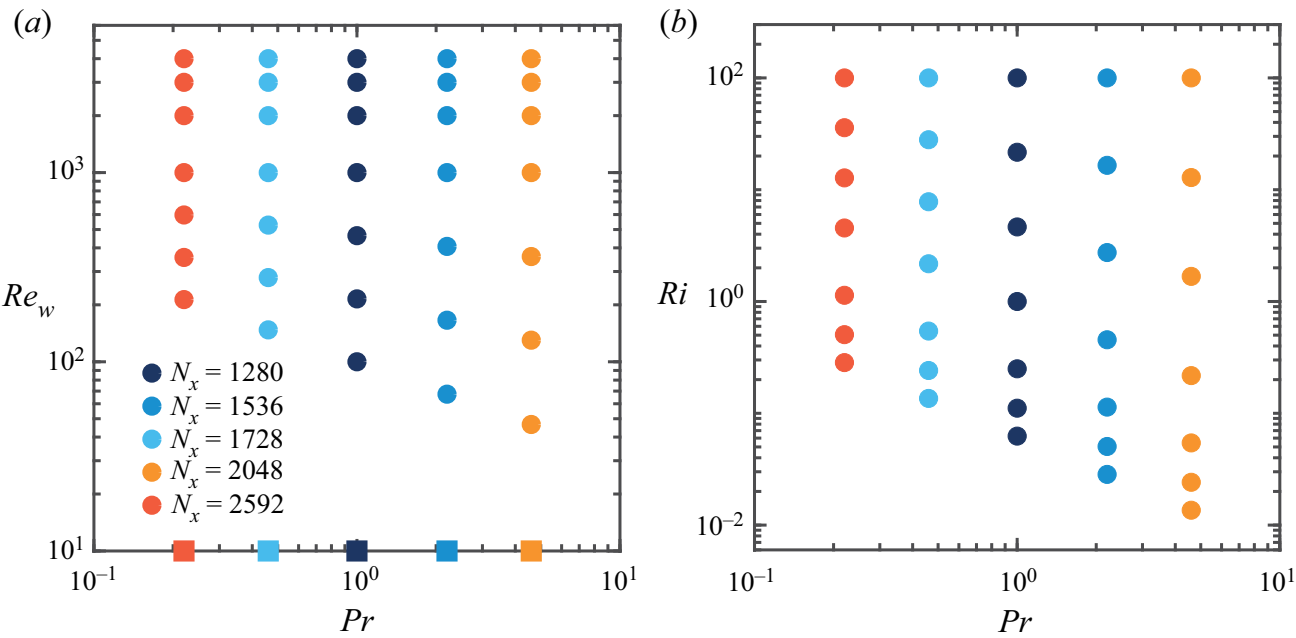

Figure 1. Phase diagram of simulation runs. We show two panels to better illustrate our choice of simulation input parameters, which were determined based on $\operatorname{Re}_{w}(a)$ and $R i(b)$. Values of $R e_{w}=2000,3000,4000$ were chosen to be consistent with Blass et al. (2020) and to cover the shear dominated regime. The squared symbols show the datapoints for $R e_{w}=0$ for completeness and independently of the $y$-axis, since they cannot be directly included in the logarithmic scale. To have a sufficient amount of data in the thermal buoyancy dominated regime, we picked $R i=100$ as the most thermal dominated case and then logarithmically spaced three more datapoints.

In both flows, Couette-RB and Poiseuille-RB, the ratio between buoyancy and mechanical forcings can be best quantified by the bulk Richardson number

$$
R i=\frac{R a}{R e_{w}^{2} P r},
$$

which is a combination of the flow governing parameters $R a, R e_{w}$ and $\operatorname{Pr}$. In the Couette-RB flow of Blass et al. (2020), Ri was used to distinguish between three different flow regimes, namely thermal buoyancy dominated, transitional and shear dominated, similarly to the case of stably stratified wall turbulence, where Zonta \& Soldati (2018) distinguish between the buoyancy dominated, buoyancy affected and turbulence dominated regimes.

Indeed, sheared stably or unstably stratified flows are present in many different situations involving both liquids and gases. Therefore the fluid properties, as reflected in the Prandtl number, play a major role (Chong et al. 2018). In the atmosphere it results in $\operatorname{Pr}=O(1)$, while in ocean dynamics $\operatorname{Pr}=O(10)$. However, a much larger $\operatorname{Pr}$ variation is found in industrial applications. For example, $P r \approx O\left(10^{-3}\right)$ for liquid metals (Teimurazov \& Frick 2017), which are for example in use for cooling applications in nuclear reactors (Usanov et al. 1999), or $\operatorname{Pr} \approx O\left(10^{3}\right)$ for molten salts or silicone oils (Vignarooban et al. 2015) for high-performance heat exchangers.

Despite this staggering range of Prandtl numbers encountered in real applications, the vast majority of studies on sheared, thermally stratified flows have been performed only at $\operatorname{Pr}=O(1)$. To overcome this limitation, in this paper we extend the work of Blass et al. (2020) for $\operatorname{Pr}=1$ by analysing the parameter space $0 \leqslant R e_{w} \leqslant 4000$ and $0.22 \leqslant \operatorname{Pr} \leqslant 4.6$ while keeping the Rayleigh number constant at $R a=10^{6}$ (see figure 1 for the complete set of simulations). 


\section{A. Blass and others}

The present study can be considered similar and complementary to that of Zhou, Taylor \& Caulfield (2017) who carried out numerical simulations with a large $\operatorname{Pr}$ variation for a stably stratified Couette flow.

The manuscript is divided in the following manner. Section 2 briefly reports the numerical method. Section 3 focusses on the global transport properties and $\S 4$ on the boundary layers. The paper ends with conclusions (§5).

\section{Numerical method}

The three-dimensional incompressible Navier-Stokes equations with the Boussinesq approximation are integrated numerically. Once non-dimensionalised, the equations read

$$
\begin{gathered}
\frac{\partial \boldsymbol{u}}{\partial t}+\boldsymbol{u} \cdot \nabla \boldsymbol{u}=-\nabla P+\left(\frac{P r}{R a}\right)^{1 / 2} \nabla^{2} \boldsymbol{u}+\theta \hat{z}, \quad \nabla \cdot \boldsymbol{u}=0, \\
\frac{\partial \theta}{\partial t}+\boldsymbol{u} \cdot \nabla \theta=\frac{1}{(\operatorname{PrR} a)^{1 / 2}} \nabla^{2} \theta,
\end{gathered}
$$

with $\boldsymbol{u}$ the velocity, normalised by $\sqrt{g \beta \Delta H}$, and $\theta$ the temperature, normalised by $\Delta ; t$ is the time normalised by $\sqrt{H /(g \beta \Delta)}$ and $P$ the pressure in multiples of $g \beta \Delta H$.

Equations (2.1 $a, b)$ and (2.2) are solved using the AFiD GPU package (Zhu et al. 2018b) which is based on a second-order finite-difference scheme (van der Poel et al. 2015). The code has been validated and verified several times (Verzicco \& Orlandi 1996; Verzicco \& Camussi 1997, 2003; Stevens, Verzicco \& Lohse 2010; Stevens, Lohse \& Verzicco 2011; Ostilla-Mónico et al. 2014; Kooij et al. 2018). We use a uniform discretisation in the horizontal periodic directions and a non-uniform mesh, with an error function-like node distribution in the wall-normal direction. To implement the sheared Couette-type forcing we move the top and bottom walls in opposite directions with velocities $\pm u_{w}$, i.e. relative velocity $2 u_{w}$ between the two plates.

Following Blass et al. (2020), we performed our simulations in a $9 \pi H \times 4 \pi H \times H$ domain, which are the streamwise, spanwise and wall-normal directions, respectively. The grid resolutions are also based on Blass et al. (2020) and then further modified to account for the Prandtl number variation in this study.

\section{Flow organisation and global transport properties}

\subsection{Organisation of turbulent structures}

Using as guideline the description of Blass et al. (2020) we observe that also in the present case the flow can be classified into buoyancy dominated, transitional and shear dominated regimes (see figure 2 and table 1 for a full overview). As shown in Blass et al. (2020), for $\operatorname{Pr}=1$ and increasing $R e_{w}$, we observe the thermal buoyancy dominated regime at $R e_{w}=0$ while already at $R e_{w}=1000,2000$ the compact thermal structures elongate into streaks and evidence the transitional regime. Further increasing the wall shear causes the streaks to meander in the spanwise direction, which indicates the shear dominated regime $\left(R e_{w}=3000,4000\right)$.

As $\operatorname{Pr}=v / \kappa$ exceeds unity, kinematic viscosity overtakes thermal diffusivity and the wall shear affects the flow structures in the bulk more easily. In fact, it can be observed that, already for $R e_{w}=1000$, the flow shows meandering behaviour of the shear dominated regime. For $\operatorname{Pr}=4.6$ and $R e_{w}=4000$ the shear is strong enough to make the effect of the thermal forcing negligible, as confirmed by the flow structures similar to the plane Couette flow. 


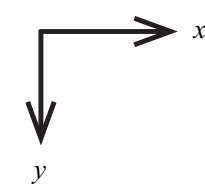

\section{$\operatorname{Pr}=0.22$}

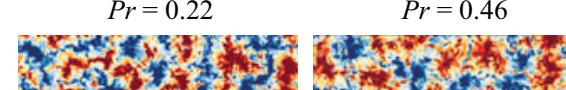

$R e_{w}=0$

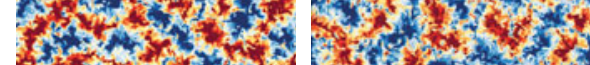

Dast

$R e_{w}=1000$

dx -1 -

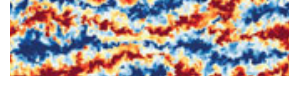

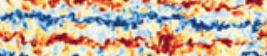

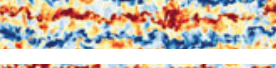

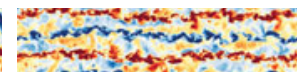

$R e_{w}=2000$

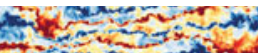

and

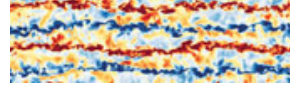

nim

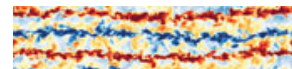

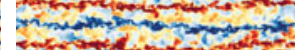

$R e_{w}=3000$

$R e_{w}=4000$

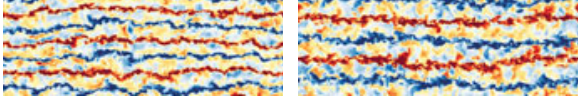

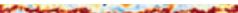

Tim

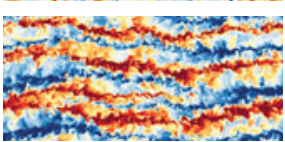

Figure 2. Snapshots of the temperature field at midheight $(z / H=0.5)$ for a subdomain of the parameter space. The applied wall shear is in the $x$-direction, while $y$ is the spanwise coordinate. 


\section{A. Blass and others}

\begin{tabular}{|c|c|c|c|c|c|c|c|c|c|c|}
\hline$R a$ & $P r$ & $R e_{w}$ & $R i$ & $N_{x}$ & $N_{y}$ & $N_{z}$ & $R e_{\tau}$ & $L_{M O} / H$ & $\mathrm{Nu}$ & $C_{f} / 10^{-3}$ \\
\hline $1.0 \times 10^{6}$ & 0.22 & 0 & $\infty$ & 2592 & 2048 & 256 & - & 0 & 7.37 & $\infty$ \\
\hline $1.0 \times 10^{6}$ & 0.22 & 213 & 100.0 & 2592 & 2048 & 256 & 57.90 & 0.006 & 7.33 & 147.5 \\
\hline $1.0 \times 10^{6}$ & 0.22 & 357 & 35.86 & 2592 & 2048 & 256 & 74.80 & 0.013 & 7.24 & 88.28 \\
\hline $1.0 \times 10^{6}$ & 0.22 & 597 & 12.76 & 2592 & 2048 & 256 & 99.81 & 0.031 & 6.98 & 55.90 \\
\hline $1.0 \times 10^{6}$ & 0.22 & 1000 & 4.546 & 2592 & 2048 & 256 & 133.0 & 0.080 & 6.44 & 35.37 \\
\hline $1.0 \times 10^{6}$ & 0.22 & 2000 & 1.137 & 2592 & 2048 & 256 & 197.0 & 0.286 & 5.89 & 19.41 \\
\hline $1.0 \times 10^{6}$ & 0.22 & 3000 & 0.505 & 2592 & 2048 & 256 & 246.7 & 0.538 & 6.14 & 13.53 \\
\hline $1.0 \times 10$ & 0.22 & 4000 & 0.284 & 2592 & 2048 & 256 & 291.5 & 0.884 & 6.17 & 10.62 \\
\hline $1.0 \times 10^{6}$ & 0.46 & 0 & $\infty$ & 1728 & 1458 & 192 & - & 0 & 7.92 & $\infty$ \\
\hline $1.0 \times 10^{6}$ & 0.46 & 147 & 100.0 & 1728 & 1458 & 192 & 43.75 & 0.005 & 7.82 & 176.1 \\
\hline $1.0 \times 10^{6}$ & 0.46 & 279 & 27.99 & 1728 & 1458 & 192 & 60.63 & 0.014 & 7.58 & 94.69 \\
\hline $1.0 \times 10^{6}$ & 0.46 & 528 & 7.803 & 1728 & 1458 & 192 & 85.08 & 0.041 & 6.98 & 51.97 \\
\hline $1.0 \times 10^{6}$ & 0.46 & 1000 & 2.175 & 1728 & 1458 & 192 & 120.2 & 0.128 & 6.26 & 28.91 \\
\hline $1.0 \times 10^{6}$ & 0.46 & 2000 & 0.544 & 1728 & 1458 & 192 & 175.0 & 0.414 & 5.96 & 15.33 \\
\hline $1.0 \times 10^{6}$ & 0.46 & 3000 & 0.241 & 1728 & 1458 & 192 & 217.8 & 0.787 & 6.04 & 10.54 \\
\hline $1.0 \times 10^{6}$ & 0.46 & 4000 & 0.136 & 1728 & 1458 & 192 & 260.7 & 1.287 & 6.33 & 8.493 \\
\hline $1.0 \times 10^{6}$ & 1 & 0 & $\infty$ & 1280 & 1024 & 256 & - & 0 & 8.34 & $\infty$ \\
\hline $1.0 \times 10^{6}$ & 1 & 100 & 100.0 & 1280 & 1024 & 128 & 31.85 & 0.004 & 8.20 & 202.9 \\
\hline $1.0 \times 10^{6}$ & 1 & 215 & 21.63 & 1280 & 1024 & 128 & 47.31 & 0.014 & 7.82 & 96.86 \\
\hline 1.0 & 1 & 464 & 4.645 & 1280 & 1024 & 128 & 72.95 & 0.056 & 6.95 & 49.44 \\
\hline $1.0 \times 10^{6}$ & 1 & 1000 & 1.000 & 1280 & 1024 & 128 & 113.5 & 0.223 & 6.56 & 25.75 \\
\hline $1.0 \times 10$ & 1 & 2000 & 0.250 & 1280 & 1024 & 256 & 161. & 0.645 & 6.56 & 13.07 \\
\hline 1.0 & 1 & 3000 & 0.111 & 1280 & 1024 & 256 & 203.0 & 1.218 & 6.87 & 9.158 \\
\hline $1.0 \times 10^{6}$ & 1 & 4000 & 0.063 & 1280 & 1024 & 256 & 251.7 & 2.022 & 7.89 & 7.922 \\
\hline $1.0 \times 10^{6}$ & 2.2 & 0 & $\infty$ & 1536 & 1296 & 162 & - & 0 & 8. & $\infty$ \\
\hline $1.0 \times$ & 2.2 & 67 & 100.0 & 1536 & 1296 & 162 & 22.88 & 0.003 & 8.38 & 230.3 \\
\hline $1.0 \times 10^{6}$ & 2.2 & 166 & 16.52 & 1536 & 1296 & 162 & 37.02 & 0.015 & 7.68 & 99.65 \\
\hline $10^{6}$ & 2.2 & 407 & 2.741 & 1536 & 1296 & 162 & 63.08 & 0.081 & 6.82 & 47.99 \\
\hline $1.0 \times 10^{6}$ & 2.2 & 1000 & 0.455 & 1536 & 1296 & 162 & 100.4 & 0.336 & 6.62 & 20.18 \\
\hline $1.0 \times 10^{6}$ & 2.2 & 2000 & 0.114 & 1536 & 1296 & 162 & 144.2 & 0.936 & 7.04 & 10.39 \\
\hline 1.0 & 2.2 & 3000 & 0.050 & 1536 & 1296 & 162 & 194.1 & 1.845 & 8.72 & 8.373 \\
\hline $1.0 \times 10^{6}$ & 2.2 & 4000 & 0.028 & 1536 & 1296 & 162 & 246.1 & 3.052 & 10.75 & 7.573 \\
\hline $1.0 \times 10^{6}$ & & ( & $\alpha$ & 20 & 5 & 192 & - & 0 & 8.5 & $\infty$ \\
\hline $1.0 \times 10^{6}$ & 4.6 & 47 & 100.0 & 2048 & 1536 & 192 & 16.68 & 0.003 & 8.31 & 255.9 \\
\hline $1.0 \times 10^{6}$ & 4.6 & 130 & 12.85 & 2048 & 1536 & 192 & 29. & 0.016 & 7.51 & 103.5 \\
\hline $1.0 \times 10^{6}$ & 4.6 & 360 & 1.678 & 2048 & 1536 & 192 & 53.01 & 0.101 & 6.77 & 43.38 \\
\hline $1.0 \times 10^{6}$ & 4.6 & 1000 & 0.217 & 2048 & 1536 & 192 & 87.65 & 0.459 & 6.75 & 15.36 \\
\hline & 4.6 & 2000 & 0.054 & 2048 & 1536 & 192 & 13 & 1.382 & 8.58 & 9.397 \\
\hline & 4.6 & 3000 & 0.024 & 2048 & 1536 & 192 & 189.0 & 2.685 & 11.56 & 7.936 \\
\hline $1.0 \times 10^{6}$ & 4.6 & 4000 & 0.014 & 2048 & 1536 & 192 & 240.4 & 4.441 & 14.39 & 7.225 \\
\hline
\end{tabular}

Table 1. Main simulations considered in this work. The columns from left to right indicate the input and output parameters and the resolution in streamwise, spanwise and wall-normal directions $\left(N_{x}, N_{y}, N_{z}\right)$. The simulations for $0 \leqslant R e_{w} \leqslant 1000$ were chosen to allow the first non-zero $R e_{w}$ at $R i=100$. The other two $R e_{w}<$ 1000 simulations for each $\operatorname{Pr}$ respectively were logarithmically evenly spaced in $R e_{w}$. Data of Blass $e t a l$. (2020) have been used for $\operatorname{Pr}=1 ; \operatorname{Re}=0,2000,3000,4000$. The data of the Monin-Obukhov length were added for consistency with Blass et al. (2020), although not specifically discussed in this manuscript. 


\section{Prandtl effects on turbulent sheared thermal convection}

Conversely, for Prandtl numbers smaller than unity, the shear is less effective for a given $R e_{w}$ and the bulk flow is more dominated by the thermal structures. In the case of $\operatorname{Pr}=$ 0.22 , a wall shear of $R e_{w}=1000$ is not strong enough to fully disturb the plumes and only the next datapoint at $R e_{w}=2000$ shows signs of elongated streaks.

From the panels of figure 2 it is evident how $\operatorname{Pr}$ changes the relative strength of the momentum and thermal diffusivities: a higher Prandtl number, corresponding to a larger kinematic viscosity, increases the momentum transfer from the boundaries to the bulk and the transition to the shear dominated regime occurs at a lower $R e_{w}$ than for a corresponding low Pr flow. Vice versa, for small Prandtl numbers, the thermal dominated regime is more persistent and the shear dominated flow features appear only at high $R e_{w}$. These findings are consistent with those of Blass et al. (2020) that the Richardson number, $R i$, which is constant for constant $\operatorname{Re}_{w}^{2} \operatorname{Pr}$ (see (1.1)), determines the flow regime.

\subsection{Heat transfer}

The Nusselt number $N u$ is plotted in figure 3 as a function of $R e_{w}$, showing a non-monotonic behaviour. The common feature is that, for increasing wall shear, $\mathrm{Nu}$ first decreases and then increases, as already observed in Blass et al. (2020) for $\operatorname{Pr}=1$. In the present case, however, the specific values are strongly dependent on $\mathrm{Pr}$, as seen in figure 3(c). The effect of $P r$ is strongly dependent on the amount of shear added to the system. For pure Rayleigh-Bénard convection $\left(R e_{w}=0\right), N u$ increases with $\operatorname{Pr}$ for $\operatorname{Pr}<1$ and saturates to a constant value for $1<\operatorname{Pr}<4.6$, see figure 3(b), in agreement with the findings of van der Poel, Stevens \& Lohse (2013) and Stevens et al. (2013). For increasing $R e_{w}$, the effect of the wall shear on the heat transfer is more pronounced for increasing $P r$, because of the higher momentum transfer from the boundaries to the bulk. This is confirmed both by the initial $\mathrm{Nu}$ decrease up to $20 \%$ of the RB value at $\operatorname{Pr}=4.6$ and the subsequent strong increase by more than $50 \%$ for the highest $R e_{w}$. In both cases the effects of the momentum transfer are enhanced by the high Prandtl number. We mention that the non-monotonic behaviour of the Nusselt number observed here is a frequently occurring feature of flows in which more than one parameter determines the value of the heat transfer; other known cases have been reported by Scagliarini et al. (2014) and Pirozzoli et al. (2017) for Poiseuille-RB flow, Yang et al. (2020) and Wang et al. (2020b) for thermal convection with rotation or Chong \& Xia (2016) for severe lateral confinement, although the exact interplays between the forces in these cases are different.

\subsection{Flow layering}

The initial $\mathrm{Nu}$ decrease can be understood upon considering that the added wall shear perturbs the thermal RB structures and produces a horizontal flow layering that weakens the vertical heat flux. Once the wall shear is strong enough, however, the flow undergoes a transition to a shear dominated regime and the vertical cross-stream motion generated by the elongated streaks makes up for the suppressed RB structures, thus starting the Nusselt number monotonic increase (Blass et al. 2020). To better understand the effect of the horizontal flow layering, we discuss the results of figure 4. In these 'side views' (i.e. streamwise cross-sections) of the temperature field snapshots and the corresponding top views of figure 2, we can observe how the flow changes from thermal plumes to straight thin streaks and then to meandering structures. As expected, the increase in wall shear causes the flow to become more turbulent. But the change in the large-scale structures is also very recognisable. Here, the transitional regime displays a more unexpected behaviour. In contrast to what is seen in figure 4(a,c), where the flow structures appear 


\section{A. Blass and others}

(a)

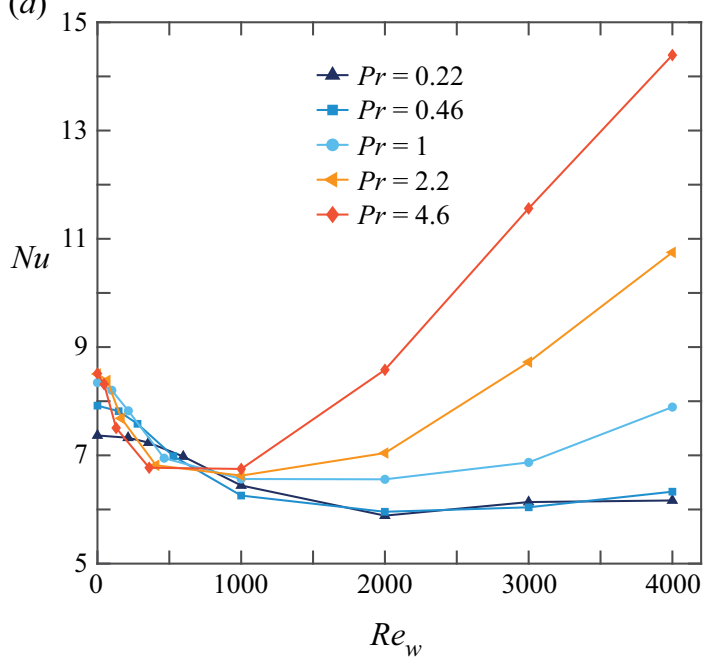

(b)

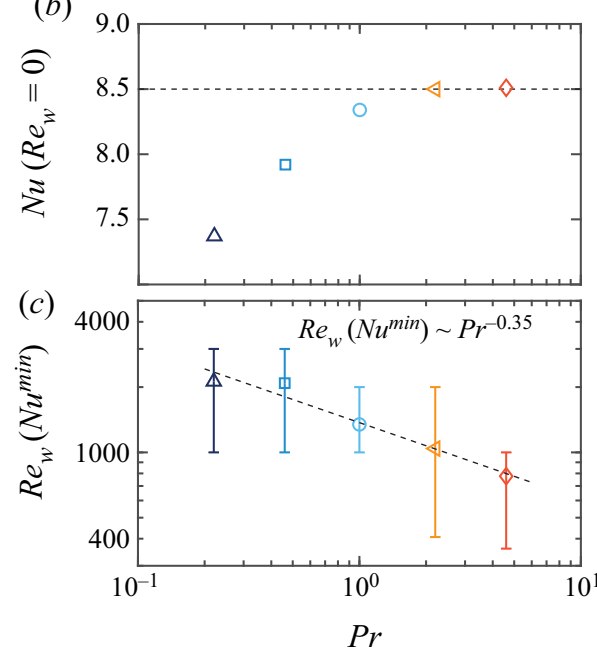

Figure 3. (a) Value of $N u$ versus $R e_{w}$ for varying $P r$. The curves show a more or less pronounced minimum $N u^{\min }$ at a certain shear Reynolds number $R e_{w}\left(N u^{\min }\right)$. (b) Shows $N u\left(R e_{w}=0\right)$ versus $\operatorname{Pr}$. (c) Shows $\operatorname{Re}_{w}\left(\mathrm{Nu}^{\mathrm{min}}\right)$ versus $\mathrm{Pr}$. Note that the error bars for these values are considerable, given our limited resolution in $R e_{w}$. Nonetheless, we include a power-law fit in the figure.

clearly divided into hot and cold columns, in figure $4(b)$ the structures are more complex. Due to the wall shear and the thereby imposed horizontal flow, the vertical structures are disturbed, the flow is not able to reach the opposite hot/cold wall, but is instead trapped in a warm/cool state in the bulk of the flow. The fluctuations in the flow are not strong enough to mix the bulk and therefore the heat gets insulated in a stably stratified layer in the middle of the flow. This layering causes the total heat transfer to decrease and is the reason for the drop in $N u$ for low $R e_{w}$ in figure 3 . Because of the heat entrapment in the bulk layer, relatively cold fluid comes very close to relatively warm fluid and the temperature gradients in the wall-normal direction increase significantly. In the atmosphere, this phenomenon can be observed as cloud streets, which, similar to the high-shear end of the transitional regime observed here, manifests as long streaks of convection rolls (Etling \& Brown 1993; Kim et al. 2003; Jayaraman \& Brasseur 2018).

\section{Boundary layers}

\subsection{Boundary layer thicknesses}

A complementary way to better understand the $P r$-dependence of the flow dynamics and the transport properties is to study the viscous and thermal boundary layer thicknesses $\lambda_{u}$ and $\lambda_{\theta}$, respectively. Here, we define both $\lambda_{\theta}$ and $\lambda_{u}$ by extrapolating the linear slopes of the mean temperature and mean streamwise velocity close to the walls, similarly to Shishkina et al. (2010). The dependence of $\lambda_{u}$ and $\lambda_{\theta}$ on $R i$ and $\operatorname{Pr}$ is shown in figure 5. Here, we use as abscissa the Richardson number. Given that $R a=10^{6}$ is constant, we have $R i \propto\left(P r R e_{w}^{2}\right)^{-1}$. At every $\operatorname{Pr}$, for increasing $R i$ - and therefore decreasing shear $-\lambda_{\theta}$ initially grows, then reaches a plateau at $R i \approx 1$ and eventually decreases slowly to converge to the pure RB value (figure $5 a$ ). For comparison, we also plot $N u(R i)$ in figure 5(c). Given that $\lambda_{\theta} \propto(\mathrm{Nu})^{-1}$ to a good approximation, the behaviour of the thermal boundary layer thickness is consistent with the Nusselt number of figures 3 and $5(c)$. 

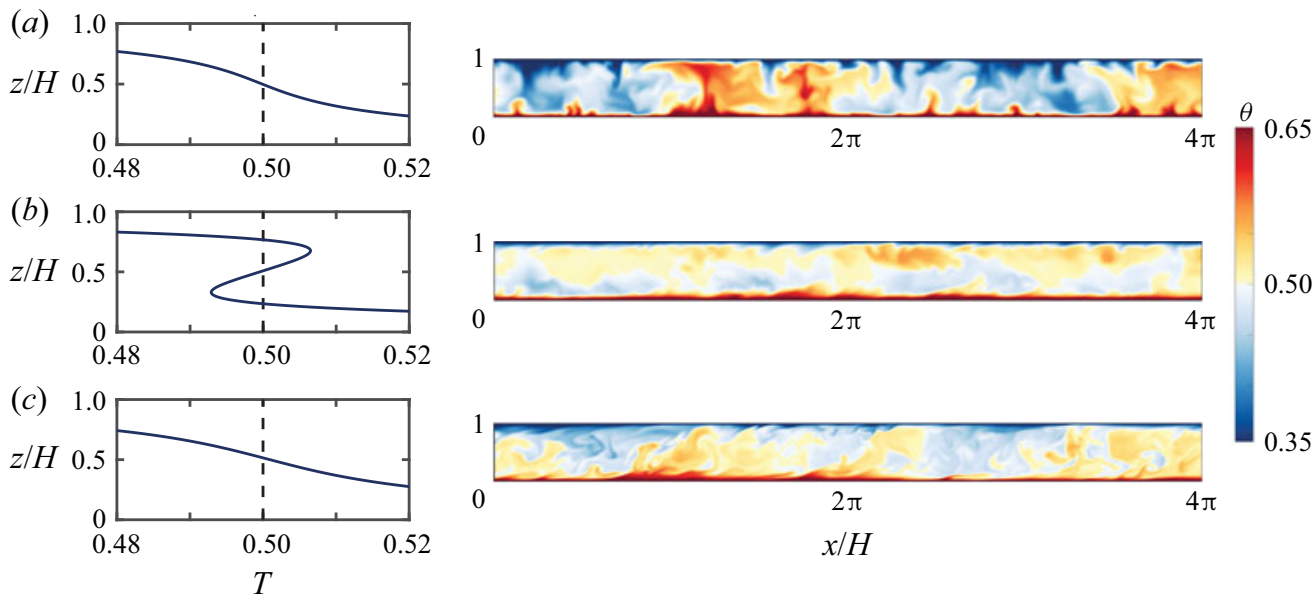

Figure 4. Mean wall-normal temperature profiles (left) and side view snapshots of temperature fields (right), i.e. streamwise cross-sections, for $(a) \operatorname{Pr}=0.22 ; \operatorname{Re}_{w}=0,(b) \operatorname{Pr}=0.22 ; \operatorname{Re}_{w}=4000$ and $(c) \operatorname{Pr}=1 ; \operatorname{Re}=$ 4000. For all right panels only $x / H=0-4 \pi$ is shown for better visibility and $y / H=2 \pi$ was chosen for the spanwise location at which periodic boundary conditions are employed.

The different flow regimes can be identified either from the different slopes of $\lambda_{\theta}$ versus $R i$ or from those of $N u(R i)$. The slope is positive in the shear dominated region (small $R i$ ), approximately zero in the transitional regime and then negative in the thermal buoyancy dominated regime.

As the Richardson number indicates the relative strength of buoyancy and shear, the non-monotonic behaviour of the thermal boundary layer can be expected. For $R i \gtrsim 1$, the flow is not dominated by shear, and therefore an increase of $R i$, which is consistent with a decrease of $R e_{w}$ for constant $R a$ and $P r$, strengthens the thermal plumes and therefore the heat transfer, which results in a smaller thermal boundary layer. The reason for the $\lambda_{\theta}$ increase for $R i \lesssim 1$ is that, in this region, the thermal forcing is weak and the flow is mainly driven by the shear. In this case the thermal boundary layer is slaved to the viscous boundary layer which, according to the expectations, monotonically thickens as the wall shear weakens. From figure $5(b)$ we can see that indeed $\lambda_{u}$ monotonically increases with increasing $R i$.

Note that the viscous boundary layer thickness has a stronger dependence on $\operatorname{Pr}$ than the thermal boundary layer thickness. Qualitatively, larger $P r$ reflects stronger momentum diffusivity and therefore a thicker viscous boundary layer. Note that part of this strong increase of $\lambda_{u}$ with $\operatorname{Pr}$ simply reflects that $R i$ is kept constant, because, to achieve this, $R e_{w}$ has to decrease as $\propto \operatorname{Pr}^{-1 / 2}$ to keep $R i$ fixed, see (1.1). However, in the shear dominated regime (high $\mathrm{Pr}$ or low $R i$ ), $\lambda_{u}$ grows faster than in the other regimes and this is especially true for the flows with higher $P r$. In fact, in these cases the thermal boundary layer is nested within the viscous one and the dynamics of the latter is not sensitive to the former. This is not the case for small $\operatorname{Pr}<1$ because then $\lambda_{u}$ evolves inside $\lambda_{\theta}$ whose thinning with increasing $R i$ counteracts the thickening of the viscous boundary layer.

To further stress the importance of the relative thicknesses of the thermal and the viscous boundary layer, we show their ratio versus $R i$ in figure $5(d)$. We can see that $\lambda_{\theta} / \lambda_{u}$ increases for decreasing $\operatorname{Pr}$ at fixed $R i$ since the kinetic boundary layer thickness is driven by the momentum diffusivity. At fixed $\operatorname{Pr}$ the behaviour of the boundary layer ratio is more complex: it always shows a decreasing trend in the high end of $R i$ which is due to 


\section{A. Blass and others}

(a)
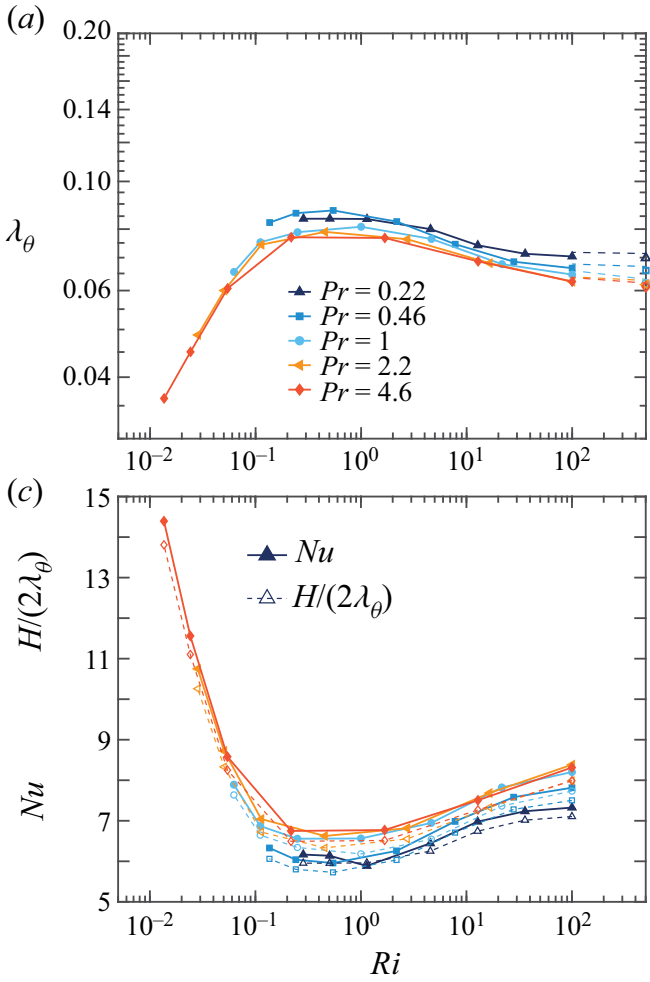

(b)
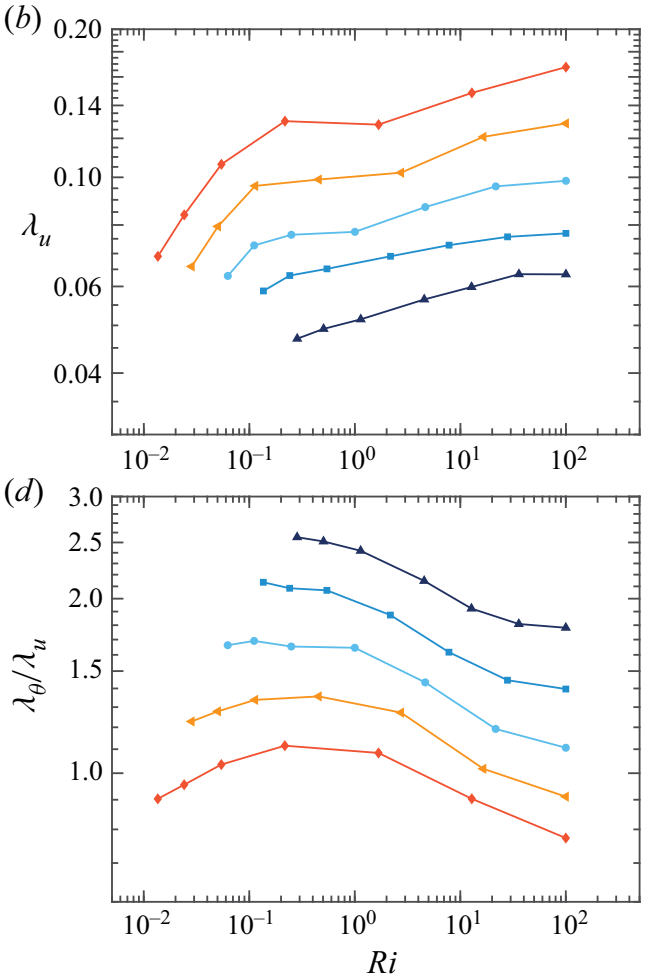

Figure 5. (a) Thermal boundary layer thickness $\lambda_{\theta}$ and $(b)$ kinetic boundary layer thickness $\lambda_{u}$ as functions of the $R i$-number for various $P r$-numbers and fixed $R a=10^{6}$. Note that the scale is the same in both $(a, b)$. (c) Value of $N u(R i)$ compared to $H /\left(2 \lambda_{\theta}(R i)\right)$. (d) Ratio of thermal and kinetic boundary layer thicknesses vs $R i$.

the thinning of the thermal boundary layer. On the other hand, at the low end of $R i$ one can observe an increase only for $\operatorname{Pr}>1$, which is due to the steep growth of $\lambda_{\theta}$ with $R i$ observed in figure $5(a)$.

Due to the limited number of datapoints, we cannot show a more detailed behaviour in the extreme case of pure shear forcing. In contrast, in the limit of pure Rayleigh-Bénard convection we do observe the asymptotic trend for $\lambda_{\theta} / \lambda_{u}$; there, the effect of the shear becomes very small (no imposed shear, all shear due to natural convection roll) and the ratio depends on $\operatorname{Pr}$ only. This saturation occurs earlier for smaller $\operatorname{Pr}$, because the thermal forcing dominates over the shear forcing at smaller $R a$.

\subsection{Velocity and temperature wall profiles}

For strong enough shear the boundary layers, which are first of laminar type, will eventually become turbulent, considerably enhancing the heat transport. However, for most of the values of the control parameters $\left(R e_{w}\right.$ and $\left.P r\right)$ of this paper this is hardly the case. This can best be judged from the velocity profiles, which we show in figure $6(a-c)$ for three different values of $\operatorname{Pr}$ and various $R e_{w}$. Only in the high- $\operatorname{Pr}$ range, towards the limit of plane Couette flow, can we see that $u^{+}$evolves towards the well-known Prandtl-von Kármán logarithmic behaviour $u^{+}\left(z^{+}\right)=\kappa^{-1} \log z^{+}+B$ for high $R e_{w}$. Since the shear strongly affects the flow, the boundary layers can undergo the transition to turbulence 
(a)

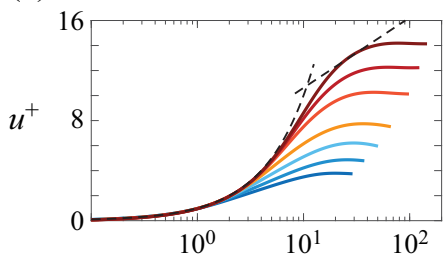

(d)

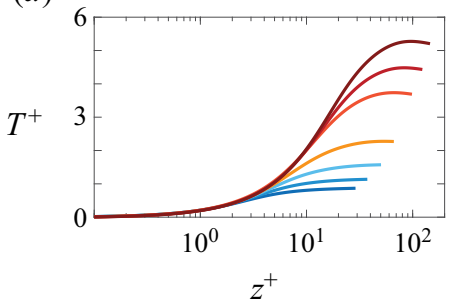

$-R e_{w}=213$

- $R e=357$

$-R e_{w}=597$

$-R e_{w}=1000$

$-R e^{w}=2000$

$-R e_{w}^{w}=3000$ (b)

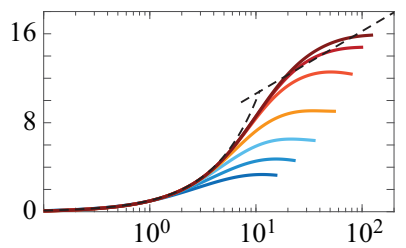

(e)

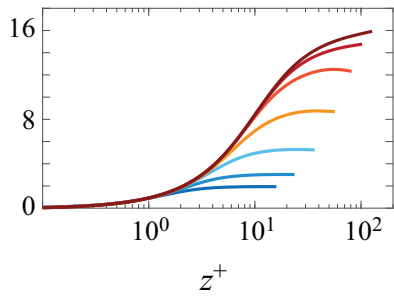

$-R e_{w}=100$

$-R e=215$

$-R e_{w}^{w}=464$

$-R e_{w}=1000$

$-R e_{w}=2000$

$-R e_{w}=3000$

$-R e_{w}=4000$ (c)

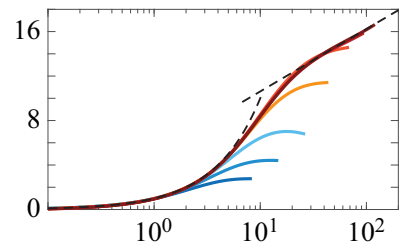

$(f)$

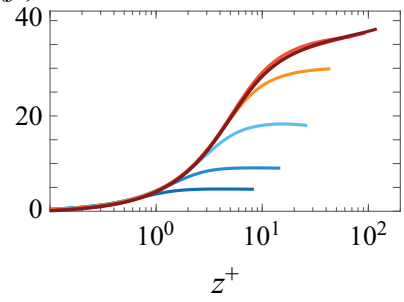

$-R e_{w}=47$

- $R e_{w}=130$

$-R e^{w}=360$

- $R e_{w}=1000$

- $R e^{w}=2000$

$-R e_{w}=3000$

$-R e_{w}=4000$

Figure 6. Velocity and temperature wall profiles for $\operatorname{Pr}=0.22$ (left), $\operatorname{Pr}=1$ (middle) and $\operatorname{Pr}=4.6$ (right) for various $R e_{w} .(a-c)$ Mean streamwise velocity and $(d-f)$ mean temperature profiles. Here, $u^{+}=u / u_{\tau}$ and $T^{+}=T / T_{\tau}$, with the friction temperature $T_{\tau}=Q / u_{\tau}$. The dashed lines in $(a-c)$ show the linear profile for $z^{+} \ll 10$ and the Prandtl-von Kármán log law of the wall $u^{+}\left(z^{+}\right)=\kappa^{-1} \log z^{+}+B$, with $\kappa=0.41$ and $B=5$.

earlier than without shear. Note that the large $\operatorname{Pr}$ number enhance the shear in the boundary layer. In fact, at $P r=4.6$ already the flow at $R e_{w}=3000$ shows the onset of a $\log$-law behaviour, in spite of the quite low $R a=10^{6}$. This occurrence of the log layer for large $P r=4.6$ goes hand in hand with an increase in the Nusselt number as a function of $R e_{w}$, see figure $3(a)$. It resembles the onset of a log-law behaviour for the velocity boundary layer profile in two-dimensional RB simulations at very large Rayleigh numbers $R a \geqslant 10^{13}$ (Zhu et al. 2018a), which also coincides with an enhanced Nusselt number and which has been associated with the onset of the ultimate regime. The same coincidence of the development of a log layer and an enhanced heat transfer had also been found by Wang, Zhou \& Sun (2020a) for high frequency horizontal vibration of the RB cell. Here, in our present simulations, the more $\mathrm{Pr}$ is decreased, the harder it becomes for the wall shear to disturb the thermal plumes and, as a result, at $R e_{w} \leqslant 4000$ and $\operatorname{Pr} \leqslant 2.2$, the log scaling cannot be attained in our simulations.

Figure $6(d-f)$ shows a similar behaviour for the mean temperature profiles as for the velocity profiles. One can observe that the temperature profiles converge earlier towards some type of logarithmic behaviour. For $\operatorname{Pr}=1$, we can see such behaviour for $\operatorname{Re}_{w}=$ 4000 , whereas at larger $P r=4.6$, it already shows up even at $R e_{w}=2000$. From the shown temperature profiles, we can also identify the flow layering that was previously discussed in $\$ 3.3$. When the flow layering occurs, heat gets entrapped in the bulk flow. Since now an additional layer of warm and cool fluid exists in between of the cold and hot regions, $T^{+}$shows a non-monotonic behaviour with a drop after the initial peak. This can most prominently be seen in figure $6(d)(P r=0.22)$ for the strongest shear $\operatorname{Re}_{w}=4000$. 


\section{A. Blass and others}

\section{Conclusion}

In this manuscript we performed direct numerical simulations of wall sheared thermal convection with $0 \leqslant R e_{w} \leqslant 4000$ and $0.22 \leqslant P r \leqslant 4.6$ at constant Rayleigh number $R a=$ $10^{6}$. Similarly to Blass et al. (2020), who analysed the $R a$-dependence of wall sheared thermal convection, we found three flow regimes and quantified them by using the bulk Richardson number and a visual analysis of two-dimensional cross-sectional snapshots. The flow undergoes a transition from the thermal buoyancy dominated to the transitional state when $R i \lesssim 10$. We found that the meandering streaks of the shear dominated regime start to emerge at $R i \lesssim 0.1$. Also the behaviour of the Nusselt number strongly depends on $\operatorname{Pr}$. For high Prandtl number, the momentum transfer from the walls to the flow is increased and therefore the flow can more easily reach the shear dominated regime where the heat transfer is again increased. We analysed both the thermal and the kinetic boundary layer thicknesses to better understand the transitions of the flow between its different regimes. We found that the thermal boundary layer thickness $\lambda_{\theta}$ shows a peak in the transitional regime and decreases for both lower and higher $R i$. The kinetic boundary layer thickness $\lambda_{u}$ increases with increasing $R i$ and increasing $P r$. For very strong $R e_{w}$ and in particular large $\operatorname{Pr}$ we notice the appearance of logarithmic boundary layer profiles, signalling the onset of turbulent boundary layer dynamics, leading to an enhanced heat transport.

Together with the results of Blass et al. (2020), we now have analysed two orthogonal cross-sections of the three-dimensional parameter space $\left(\operatorname{Ra}, \operatorname{Pr}, \operatorname{Re}_{w}\right)$. More specifically, we have determined $N u\left(R a, P r, R e_{w}\right)$ for the two cross-sections $N u\left(R a, P r=1, R_{w}\right)$ in Blass et al. (2020) and $N u\left(R a=10^{6}, P r, R e_{w}\right)$ here. From standard RB without shear we of course know $N u\left(R a, P r, R e_{w}=0\right)$, which is perfectly described by the unifying theory of thermal convection by Grossmann \& Lohse $(2000,2001)$ and Stevens et al. (2013). The knowledge of the two new cross-sections in parameter space may enable us to extend this unifying theory to sheared convection.

Acknowledgements. We thank P. Berghout, K.L. Chong and O. Shishkina for fruitful discussions. The simulations were supported by a grant from the Swiss National Supercomputing Centre (CSCS) under project ID s713, s802, and s874. This work was financially supported by NWO and the Priority Programme SPP 1881 'Turbulent Superstructures' of the Deutsche Forschungsgemeinschaft. We also acknowledge the Dutch national e-infrastructure SURFsara with the support of SURF cooperative.

Declaration of interests. The authors report no conflict of interest.

Author ORCIDs.

(1) Alexander Blass https://orcid.org/0000-0003-1618-1873;

Roberto Verzicco https://orcid.org/0000-0002-2690-9998;

Richard J.A.M. Stevens https://orcid.org/0000-0001-6976-5704;

Detlef Lohse https://orcid.org/0000-0003-4138-2255.

\section{REFERENCES}

Ahlers, G., Grossmann, S. \& Lohse, D. 2009 Heat transfer and large scale dynamics in turbulent Rayleigh-Bénard convection. Rev. Mod. Phys. 81, 503-537.

BARKLEY, D. \& TUCKERMAN, L.S. 2005 Computational study of turbulent laminar patterns in Couette flow. Phys. Rev. Lett. 94 (1), 014502.

Blass, A., Zhu, X., Verzicco, R., Lohse, D. \& Stevens, R.J.A.M. 2020 Flow organization and heat transfer in turbulent wall sheared thermal convection. J. Fluid Mech. 897, A22.

Chantry, M., Tuckerman, L.S. \& BARKLEy, D. 2017 Universal continuous transition to turbulence in a planar shear flow. J. Fluid Mech. 824, R1.

Chilla, F. \& Schumacher, J. 2012 New perspectives in turbulent Rayleigh-Bénard convection. Eur. Phys. J. E 35, 58. 


\section{Prandtl effects on turbulent sheared thermal convection}

Chong, K.L., Wagner, S., Kaczorowski, M., Shishkina, O. \& XiA, K.-Q. 2018 Effect of Prandtl number on heat transport enhancement in Rayleigh-Bénard convection under geometrical confinement. Phys. Rev. Fluids 3, 013501.

Chong, K.L. \& XiA, K.-Q. 2016 Exploring the severely confined regime in Rayleigh-Bénard convection. J. Fluid Mech. 805, R4.

DEARDORFF, J.W. 1972 Numerical investigation of neutral and unstable planetary boundary layers. J. Atmos. Sci. 29 (1), 91-115.

DomaradzKi, J.A. \& MetCAlfe, R.W. 1988 Direct numerical simulations of the effects of shear on turbulent Rayleigh-Bénard convection. J. Fluid Mech. 193, 499.

ETLING, D. \& BROWN, R.A. 1993 Roll vortices in the planetary boundary layer: a review. Boundary-Layer Meteorol. 65, 215-248.

FUKUi, K. \& NAKAJimA, M. 1985 Unstable stratification effects on turbulent shear flow in the wall region. Intl J. Heat Mass Transfer 28, 2343-2352.

Grossmann, S. \& Lohse, D. 2000 Scaling in thermal convection: a unifying view. J. Fluid Mech. 407, 27-56.

Grossmann, S. \& Lohse, D. 2001 Thermal convection for large Prandtl number. Phys. Rev. Lett. 86, 3316-3319.

Hathaway, D. \& Somerville, R. 1986 Nonlinear interactions between convection, rotation and flows with vertical shear. J. Fluid Mech. 164, 91-105.

IngERSOLL, A.P. 1966 Thermal convection with shear at high Rayleigh number. J. Fluid Mech. 25, $209-228$.

JAYARAMAN, B. \& BRASSEUR, J.G. 2018 The surprising transition in atmospheric boundary layer turbulence structure from neutral to moderately convective stability states and mechanisms underlying large-scale rolls. arXiv: $1807.03336 \mathrm{v} 2$.

KhannA, S. \& Brasseur, J.G. 1998 Three-dimensional buoyancy- and shear-induced local structure of the atmospheric boundary layer. J. Atmos. Sci. 55 (5), 710-743.

KIM, S.-W., PARK, S.-U. \& MoEnG, C.-H. 2003 Entrainment processes in the convective boundary layer with varying wind shear. Boundary-Layer Meteorol. 108, 221-245.

Kiтон, O. \& UMEKi, M. 2008 Experimental study on large-scale streak structure in the core region of turbulent plane Couette flow. Phys. Fluids 20, 025107.

Kooij, G.L., Botchev, M.A., Frederix, E.M.A., Geurts, B.J., Horn, S., Lohse, D., van der Poel, E.P., Shishkina, O., Stevens, R.J.A.M. \& Verzicco, R. 2018 Comparison of computational codes for direct numerical simulations of turbulent Rayleigh-Bénard convection. Comput. Fluids 166, 1-8.

LeE, M. \& Moser, R.D. 2018 Extreme-scale motions in turbulent plane Couette flows. J. Fluid Mech. 842, $128-145$.

LeE, M.J. \& Kim, J. 1991 The structure of turbulence in a simulated plane Couette flow. In Proceedings of the 8th Symposium on Turbulent Shear Flows, Munich, pp. 5.3.1-5.3.6.

LohsE, D. \& XIA, K.-Q. 2010 Small-scale properties of turbulent Rayleigh-Bénard convection. Annu. Rev. Fluid Mech. 42, 335-364.

MoEnG, C.-H. 1984 A large-eddy-simulation model for the study of planetary boundary-layer turbulence. J. Atmos. Sci. 41 (13), 2052-2062.

Orlandi, P., Bernardini, M. \& Pirozzoli, S. 2015 Poiseuille and Couette flows in the transitional and fully turbulent regime. J. Fluid Mech. 770, 424-441.

Ostilla-Mónico, R., van der Poel, E.P., Verzicco, R., Grossmann, S. \& Lohse, D. 2014 Exploring the phase diagram of fully turbulent Taylor-Couette flow. J. Fluid Mech. 761, 1-26.

Pirozzoli, S., BERnARdini, M. \& ORLANDi, P. 2011 Large-scale motions and inner/outer layer interactions in turbulent Couette-Poiseuille flows. J. Fluid Mech. 680, 534-563.

Pirozzoli, S., Bernardini, M. \& ORlandi, P. 2014 Turbulence statistics in Couette flow at high Reynolds number. J. Fluid Mech. 758, 327-343.

Pirozzoli, S., Bernardini, M., VerzicCo, R. \& Orlandi, P. 2017 Mixed convection in turbulent channels with unstable stratification. J. Fluid Mech. 821, 482-516.

VAn Der Poel, E.P., Ostilla-Mónico, R., Donners, J. \& Verzicco, R. 2015 A pencil distributed finite difference code for strongly turbulent wall-bounded flows. Comput. Fluids 116, 10-16.

VAn Der Poel, E.P., Stevens, R.J.A.M. \& Lohse, D. 2013 Comparison between two and three dimensional Rayleigh-Bénard convection. J. Fluid Mech. 736, 177-194.

Scagliarini, A., Einarsson, H., Gylfason, A. \& Toschi, F. 2015 Law of the wall in an unstably stratified turbulent channel flow. J. Fluid Mech. 781, R5.

Scagliarini, A., Gylfason, A. \& Toschi, F. 2014 Heat-flux scaling in turbulent Rayleigh-Bénard convection with an imposed longitudinal wind. Phys. Rev. E 89, 043012. 


\section{A. Blass and others}

Shishinan, O., Stevens, R.J.A.M., Grossmann, S. \& Lohse, D. 2010 Boundary layer structure in turbulent thermal convection and its consequences for the required numerical resolution. New J. Phys. 12, 075022.

Solomon, T.H. \& Gollub, J.P. 1990 Sheared boundary layers in turbulent Rayleigh-Bénard convection. Phys. Rev. Lett. 64, 2382-2385.

Stevens, R.J.A.M., Lohse, D. \& VerzicCO, R. 2011 Prandtl and Rayleigh number dependence of heat transport in high Rayleigh number thermal convection. J. Fluid Mech. 688, 31-43.

Stevens, R.J.A.M., VAn der Poel, E.P., Grossmann, S. \& Lohse, D. 2013 The unifying theory of scaling in thermal convection: the updated prefactors. J. Fluid Mech. 730, 295-308.

Stevens, R.J.A.M., Verzicco, R. \& Lohse, D. 2010 Radial boundary layer structure and Nusselt number in Rayleigh-Bénard convection. J. Fluid Mech. 643, 495-507.

Teimurazov, A. \& Frick, P. 2017 Thermal convection of liquid metal in a long inclined cylinder. Phys. Rev. Fluids 2, 113501.

Thurlow, E.M. \& Klewicki, J.C. 2000 Experimental study of turbulent Poiseuille-Couette flow. Phys. Fluids 12, 865-875.

Tsukahara, T., Kawamura, H. \& Shingai, K. 2006 DNS of turbulent Couette flow with emphasis on the large-scale structure in the core region. J. Turbul. 7, N19.

Tuckerman, L.S. \& Barkley, D. 2011 Patterns and dynamics in transitional plane Couette flow. Phys. Fluids 23 (4), 041301.

Usanov, V.I., Pankratov, D.V., Popov, É.P., Markelov, P.I., Ryabaya, L.D. \& Zabrodskaya, S.V. 1999 Long-lived radionuclides of sodium, lead-bismuth, and lead coolants in fast-neutron reactors. Atom. Energy 87, 658-662.

VERZICCO, R. \& CAMUSSI, R. 1997 Transitional regimes of low-Prandtl thermal convection in a cylindrical cell. Phys. Fluids 9, 1287-1295.

VERZICCO, R. \& CAMUSSI, R. 2003 Numerical experiments on strongly turbulent thermal convection in a slender cylindrical cell. J. Fluid Mech. 477, 19-49.

VERZICCO, R. \& ORLANDI, P. 1996 A finite-difference scheme for three-dimensional incompressible flow in cylindrical coordinates. J. Comput. Phys. 123, 402-413.

Vignarooban, K., Xu, X., Arvay, A., Hsu, K. \& Kannan, A.M. 2015 Heat transfer fluids for concentrating solar power systems - a review. Appl. Energy 146, 383-396.

WANG, B.-F., Zhou, Q. \& Sun, C. 2020 a Vibration-induced boundary-layer destabilization achieves massive heat-transport enhancement. Sci. Adv. 6 (21), eaaz8239.

Wang, Q., Chong, K.L., Stevens, R.J.A.M., Verzicco, R. \& Lohse, D. $2020 b$ From zonal flow to convection rolls in Rayleigh-Bénard convection with free-slip plates. J. Fluid Mech. 905, A21.

XIA, K.-Q. 2013 Current trends and future directions in turbulent thermal convection. Theor. Appl. Mech. Lett. 3, 052001 .

YAng, Y., Verzicco, R., Lohse, D. \& SteVens, R.J.A.M. 2020 What rotation rate maximizes heat transport in rotating Rayleigh-Bénard convection with prandtl number larger than one? Phys. Rev. Fluids 5, 053501 .

Zhou, Q., Taylor, J.R. \& CAulfield, C.P. 2017 Self-similar mixing in stratified plane Couette flow for varying Prandtl number. J. Fluid Mech. 820, 86-120.

Zhu, X., Mathai, V., Stevens, R.J.A.M., Verzicco, R. \& Lohse, D. $2018 a$ Transition to the ultimate regime in two-dimensional Rayleigh-Bénard convection. Phys. Rev. Lett. 120, 144502.

Zhu, X., Phillips, E., Arza, V.S., Donners, J., Ruetsch, G., Romero, J., Ostilla-Mónico, R., YAng, Y., Lohse, D., Verzicco, R., FAticA, M. \& Stevens, R.J.A.M. 2018b AFiD-GPU: a versatile Navier-Stokes solver for wall-bounded turbulent flows on GPU clusters. Comput. Phys. Commun. 229 , 199-210.

ZontA, F. \& Soldati, A. 2018 Stably stratified wall-bounded turbulence. Appl. Mech. Rev. 70, 040801. 